\title{
L'evoluzione degli studi di management control: un percorso nel segno della varietà
}

\author{
Maria Serena Chiucchi e Marco Gatti*
}

Varietà. È questa una delle possibili chiavi di lettura dell'evoluzione conosciuta dagli studi di management control. A cinquant'anni dal lavoro di Anthony (1965), le traiettorie di ricerca tracciate dagli Studiosi sono state molteplici e hanno investito vari aspetti del controllo, conducendo ad un graduale e progressivo ampliamento dei confini di una disciplina che sembra essere nel pieno del suo percorso evolutivo.

È una varietà, quella richiamata, che investe temi, approcci e contesti. Dal lato dei temi, è rimarcabile l'intenzione di ampliare la prospettiva di analisi del management control estendendone l'osservazione oltre la tradizionale visione di sistema a supporto delle decisioni attinenti alla gestione operativa. L'affermazione di filoni di studio sul controllo strategico, sul controllo organizzativo, sul capitale intellettuale, su ICT, business analytics e big data e sulle funzioni e sul ruolo del controller testimonia la tensione verso un'interpretazione sempre più ampia del ruolo e delle funzioni ascrivibili al sistema di controllo. Interpretazione che ha condotto al rapido proliferare di soluzioni informative, contabili e/o extra-contabili, che hanno, a loro volta, favorito l'avvio di nuovi filoni di studio focalizzati sui benefici e sulle criticità scaturenti dall'implementazione delle stesse oltre che sugli impatti organizzativi derivanti dal loro utilizzo.

Dal lato degli approcci, la varietà degli studi trova conferma nella molteplicità di metodologie e metodi di ricerca impiegati. Sia in ambito nazionale che internazionale, ricerche qualitative e quantitative hanno offerto prospettive di osservazione del management control diverse, suffragando l'idea che una disciplina tanto poliedrica non possa che essere esplorata ricorrendo a metodologie e metodi differenziati. Pur nella sostanziale varietà degli approcci metodologici seguiti, che rappresenta comunque un fattore

* Dipartimento di Management, Università Politecnica delle Marche. E-mail: m.s.chiucchi@univpm.it,m.gatti@univpm.it. 
di ricchezza per gli studi di management control, forte è la tendenza a convergere verso lo sviluppo di ricerche empiriche. L'osservazione e l'interpretazione della realtà, infatti, sono condizioni pressoché irrinunciabili per discipline, come il management control, che affondano le proprie radici nella prassi e che, nella prassi, trovano spunti per supportare nuove elaborazioni teoriche. La ricerca empirica diviene, dunque, uno strumento centrale per contribuire a colmare il gap tra teoria e prassi.

Infine, è dal lato dei contesti analizzati che la varietà degli studi di management control trova ulteriore conferma. Nel tempo, infatti, le peculiarità caratterizzanti il contesto hanno assunto un ruolo sempre più centrale per l'interpretazione del modo in cui i sistemi di controllo sono implementati ed operano. Spesso, gli studi hanno fatto riferimento a specifiche fattispecie aziendali, esplorando l'impatto delle relative caratteristiche distintive sull'implementazione, l'utilizzo e il funzionamento di sistemi di controllo. È il caso delle aziende familiari e delle piccole e medie aziende, realtà portanti del nostro tessuto imprenditoriale, che hanno da sempre suscitato l'interesse degli Studiosi, sia sul piano teorico sia su quello empirico. È anche il caso delle aziende pubbliche e di quelle sanitarie che, nel corso degli anni, hanno offerto importanti spunti di riflessione in merito all'influenza del contesto aziendale sulla configurazione e sull'uso di sistemi di controllo.

Accanto a questa accezione di contesto, prevalentemente riconducibile all'uniformità di caratteri distintivi riferibili a specifiche fattispecie aziendali, gli Studiosi hanno proposto interpretazioni alternative in cui i confini del contesto di analisi tendono a restringersi, in alcuni casi, e ad ampliarsi, in altri. Tra le prime rientrano quegli studi che, interpretando i sistemi di controllo come social practices, hanno esplorato "how accounting might be shaped by its context but also how at least some aspects of that very same context might in turn be shaped by accounting itself” (Hopwood, 1983, p. 298). In questa visione, è lo specifico contesto aziendale a rappresentare lo scenario nell'ambito del quale analizzare il funzionamento dei sistemi di controllo. Tra le seconde rientrano, invece, quelle ricerche che hanno compiuto analisi a livello internazionale inerenti al grado di implementazione e di diffusione degli strumenti di controllo.

Questo numero di Management Control accoglie contributi che sono esempi di varietà nelle diverse direzioni richiamate. Essi, infatti, testimoniano la varietà di temi e di approcci metodologici che ha caratterizzato il percorso evolutivo degli studi di management control. Gli articoli hanno in comune, in particolare, il fatto di essere tutti studi empirici condotti, in pre- 
valenza, nel contesto delle piccole e medie imprese. La varietà dei temi si coniuga, quindi, con una tendenziale omogeneità dei contesti analizzati offrendo, così, nuove prospettive di analisi di particolare interesse sia da un punto di vista teorico sia empirico.

Il primo contributo di Lucrezia Songini e Paola Vola analizza il ruolo dei processi di professionalizzazione e di managerializzazione nel contesto delle aziende familiari. Particolare enfasi è posta sull'influenza di tali processi in uno dei momenti che la letteratura e la prassi individuano come tra i più critici, quello della successione. Attraverso uno studio di caso longitudinale, condotto presso una media impresa, le Autrici analizzano la relazione esistente tra managers che non sono parte della famiglia e l'adozione di sistemi di controllo manageriale offrendo interessanti evidenze sul ruolo giocato da questi ultimi rispetto ai processi di successione.

Anche il contributo di Giovanni Bronzetti e di Romilda Mazzotta si basa su una ricerca empirica condotta nel contesto di una piccola impresa familiare. L'analisi, però, verte sulle problematiche inerenti al trasferimento del capitale intellettuale, in particolar modo di quello umano e relazionale, nel momento del passaggio generazionale. Gli Autori sviluppano un'analisi critica delle problematiche che emergono durante la fase di successione imprenditoriale ponendo in evidenza il ruolo fondamentale giocato dal trasferimento di conoscenze e di relazioni già acquisite per garantire la buona riuscita di una così complessa fase della vita delle piccole aziende familiari.

Il contributo di Francesca Maria Cesaroni e di Domenico Consoli mantiene la focalizzazione sul contesto delle piccole imprese ma sposta l'attenzione sull'analisi del livello di diffusione dell'ICT, aspetto della dimensione statica del controllo che ha acquisito crescente rilevanza nel corso degli ultimi anni. La peculiarità di questo studio è lo sviluppo di un utile strumento, il Cubo della Predisposizione Tecnologica Aziendale, per determinare il posizionamento delle piccole imprese con riferimento all'ICT, considerando anche la dimensione interattiva, non contemplata dai framework precedentemente elaborati. L'articolo propone anche un'applicazione dello strumento su un campione di piccole imprese che presentano livelli di diffusione dell'ICT diversi e che, pertanto, consentono una completa e dettagliata analisi delle diverse posizioni individuabili nell'ambito del Cubo.

Il contributo di Lorenzo Lucianetti e di Valentina Battista esplora un tema di particolare interesse: il ruolo giocato dalla pressione del management e dei tratti personali del controller rispetto alle manipolazioni dei valori di bilancio. Attraverso un'analisi quantitativa, gli Autori analizzano l'impatto del disimpegno morale e dell'impegno professionale del control- 
ler, oltre che delle pressioni esercitate dal Top Management, sulla propensione a manipolare i valori di bilancio. La ricerca condotta si colloca nel novero degli studi che affrontano il tema del controller e del suo ruolo distinguendosi, però, adottando una prospettiva di analisi più focalizzata sui tratti personali dello stesso.

Infine, questo numero di Management Control accoglie il contributo di Marco Giuliani e Stefano Marasca. L'articolo si colloca nell'ambito del vivace e quanto mai attuale dibattito sulla valutazione della ricerca, tema particolarmente caro alla nostra Rivista che, già in passato, ha accolto contributi a questo riguardo. In particolare, gli Autori approfondiscono caratteristiche, aspetti a favore e contro i vari indicatori citazionali e propongono interessanti spunti di riflessione sull'applicabilità di tali indici per la valutazione della ricerca nell'ambito delle discipline economico-aziendali.

\section{Bibliografia}

Anthony R.N. (1965), Planning and control systems: framework for analysis, Boston, Graduate School of Business Administration.

Hopwood A.G. (1983), On trying to study accounting in the context in which it operates, Accounting, Organizations and Society, 8, 2-3, pp. 287-305.

Marchi L. (2011), L'evoluzione del controllo di gestione nella prospettiva informativa e gestionale esterna, Management Control, 3, pp. 5-16. 